\title{
Getting the right stuff: Controlling neural stem cell state and fate in vivo and in vitro with biomaterials
}

\author{
Ana I Teixeira ${ }^{1}$, Joshua K Duckworth ${ }^{1}$, Ola Hermanson ${ }^{1}$ \\ ${ }^{1}$ Center of Excellence in Developmental Biology (CEDB), Organic Bioelectronics (OBOE), Department of Neuroscience, Karolinska \\ Institutet, SE17177 Stockholm, Sweden
}

Stem cell therapy holds great promises in medical treatment by, e.g., replacing lost cells, re-constitute healthy cell populations and also in the use of stem cells as vehicles for factor and gene delivery. Embryonic stem cells have rightfully attracted a large interest due to their proven capacity of differentiating into any cell type in the embryo in vivo. Tissue-specific stem cells are however already in use in medical practice, and recently the first systematic medical trials involving human neural stem cell (NSC) therapy have been launched. There are yet many obstacles to overcome and procedures to improve. To ensure progress in the medical use of stem cells increased basic knowledge of the molecular mechanisms that govern stem cell characteristics is necessary. Here we provide a review of the literature on NSCs in various aspects of cell therapy, with the main focus on the potential of using biomaterials to control NSC characteristics, differentiation, and delivery. We summarize results from studies on the characteristics of endogenous and transplanted NSCs in rodent models of neurological and cancer diseases, and highlight recent advancements in polymer compatibility and applicability in regulating NSC state and fate. We suggest that the development of specially designed polymers, such as hydrogels, is a crucial issue to improve the outcome of stem cell therapy in the central nervous system.

Cell Research (2007) 17:56-61. doi:10.1038/sj.cr.7310141; published online 9 January 2007

Keywords: transplantation, cell therapy, neurons, oligodendrocytes, polymers, biodegradable, nanofibers

\section{Introduction}

Cell types associated with the nervous system, namely neurons, astrocytes and oligodendrocytes, all originate from a single class of progenitor cells, called neural stem cells (NSCs). NSCs have been isolated from both the developing central nervous system (CNS) and peripheral nervous system (PNS) [1-3]. During CNS development NSCs line the neural tube and sequentially give rise to populations of neurons, astrocytes and finally oligodendrocytes [4]. In the developing PNS, cells delaminating from the dorsal most region of the neural tube, maintain the ability to self renew and give rise not only to cells of neural lineages, but

\footnotetext{
Correspondence: Ana I Teixeira ${ }^{1}$ and Ola Hermanson ${ }^{2}$

${ }^{1,2}$ Tel: +46-8-5248-7791, Fax: +46-8-324-927.

${ }^{1}$ E-mail: Ana.Teixeira@cmb.ki.se

${ }^{2}$ E-mail: Ola.Hermanson@ki.se
}

in addition can differentiate to mesenchymal derivatives [5]. NSCs have also been isolated from the adult CNS, dispelling the notion that the adult brain was incapable of plastic change at a cellular level $[6,7]$.

Much interest has been focused on how the extrinsic environment influences NSC fate choice. In vitro NSCs, derived from the CNS, have been shown to respond to the addition of single extrinsic determinants and differentiate to specific neural and mesenchymal lineages (Figure 1). Though this has helped elucidating many of the cell's intrinsic mechanisms involved in NSC differentiation, including both signaling and epigenetic pathways, it fails to reflect the broad spectrum of signaling factors known to converge on these cells in vivo. Recently the use of biomaterials has begun to reveal new ways to investigate what controls NSC fate choice [8,9]. This is an important first step, as it gets researchers closer to the goal of integrating NSCs into the broad range of envisioned stem cell therapies. In this review 

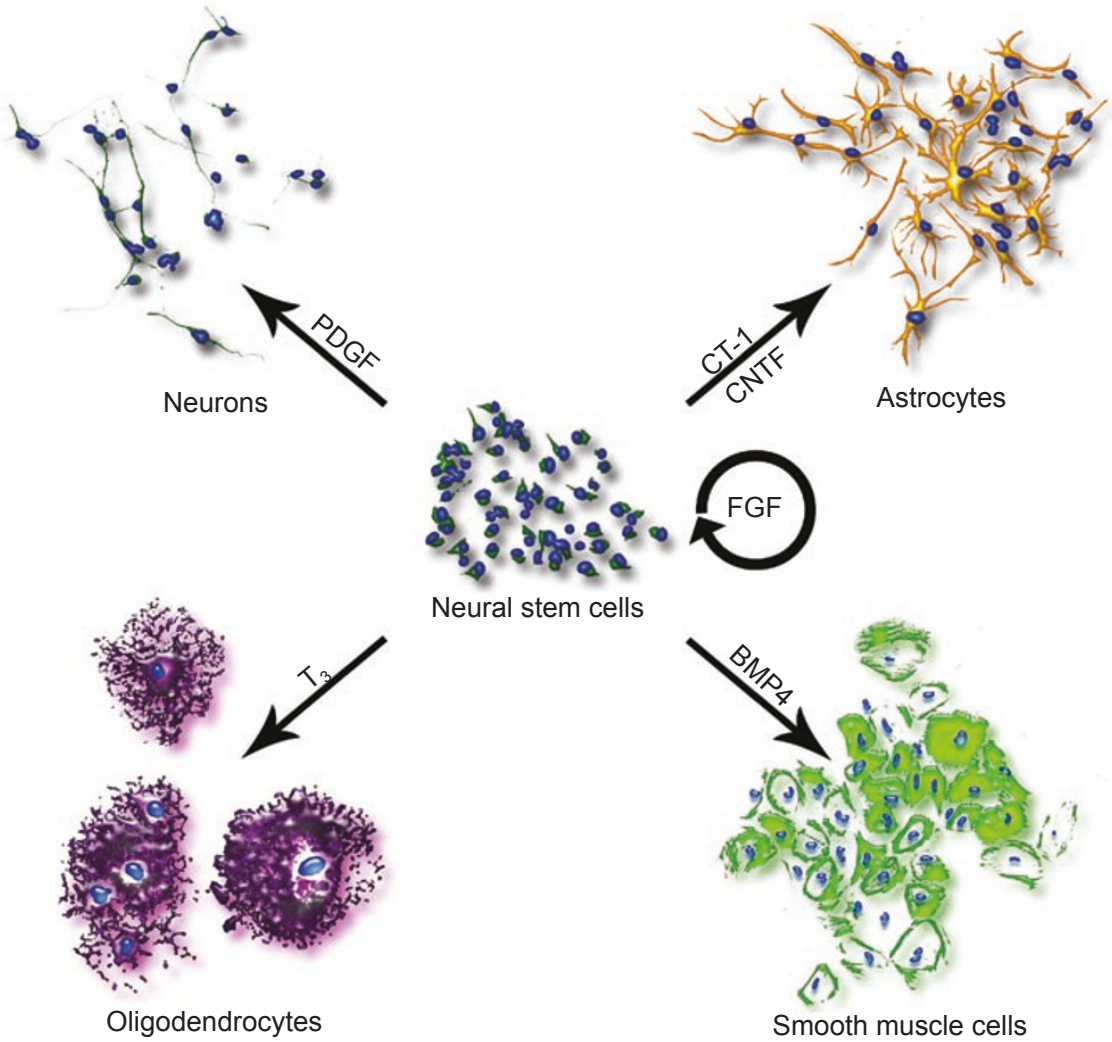

Figure 1 NSCs isolated from the developing forebrain can differentiate to different cell fates in vitro upon use of single factors supplemented in the growth media. Stem cell characteristics such as self-renewal and differentiation potential of NSCs are maintained by fibroblast growth factor (FGF). Neuronal and astrocytic differentiation can be induced by platelet derived growth factor (PDGF) and cardiotrophin-1 (CT-1) / cilliary neurotrophic factor (CNTF) respectively [46-48]. Oligodendrocytic differentiation can be induced by thyroid hormone $\left(\mathrm{T}_{3}\right)$, and in addition NSCs can also differentiate to mesenchymal cell fates by stimulation with bone morphogenic protein-4 (BMP4) [47, 49].

we will focus on recent advances in this area and discuss how material science is pointing the way forward in our understanding of NSC state and fate, and how biomaterials may be of potential use in implementing NSCs into future stem cell therapy.

\section{Neural stem cells as multipurpose tools in cell therapy}

The potential for controlled differentiation into all neural tissues makes NSCs very attractive for regeneration therapy in the nervous system. Additional, and perhaps unexpected, properties of NSCs expand their potential use as therapeutic tools. These include the capacity to home in on injury or inflammation sites and to provide trophic support to remaining healthy tissues.

\section{Using exogenous NSCs in cell replacement therapy}

Neural stem cells can potentially be used to replace tissue loss due to trauma or neurodegenerative diseases. Ideally, the precise architecture of the tissue should be reconstructed and integrated with surrounding tissues. Importantly, several neural cell types need to be replaced in this scenario. NSCs derived in vitro from human embryonic stem cells [10] or isolated from human fetal brain [11], when transplanted into the adult rodent brain, differentiated into neurons, astrocytes and oligodendrocytes. Notably, NSCs integrated into the neurogenic niche of the subventricular zone and contributed to neurogenesis in the olfactory bulb. In the hippocampus, transplanted neural progenitors were observed to differentiate into regionspecific neuronal subtypes and functionally integrate into the local circuitry [12].

NSC transplantation into disease or injury rodent models has largely presented promising results. Transplanted NSCs have been reported to survive, migrate primarily towards the pathological site, and differentiate. Adult mouse NSCs transplanted into a mouse model of multiple 
sclerosis caused an increase in the numbers of oligodendrocyte progenitor and mature cells that are of both the host and transplant origin, resulting in a remarkable recovery from the disease [13]. In a rat model of brain ischemia, transplanted human NSCs migrated to the injury and differentiated mostly into neurons [14]. NSCs transplanted intravenously in a model of intracerebral hemorrhage differentiated into neurons and astrocytes at the lesion site, resulting in functional recovery [15]. Neuronal differentiation and functional recovery were reported following transplantation of NSCs derived from the rat embryonic spinal cord into a model of contusion spinal cord injury (SCI) [16]. Improved motor function was also reported in a model of weight-drop SCI after transplantation of NSCs retrieved from adult rats [17]. NSCs differentiated mostly into astrocytes and oligodendrocytes but these animals showed aberrant axonal sprouting at the injury site and allodynia-like hypersensitivity of forepaws. Allodynia was also observed after transplantation of neural progenitor-like C17.2 cells into the injured spinal cord [18].

\section{Injury-induced recruitment of endogenous NSCs}

The presence of stem cell niches in multiple regions of the CNS in the adult has raised the hypothesis that NSCs may be naturally called into action during pathological states. Tracing of nestin positive neural progenitors resident in the adult spinal cord has revealed that these cells proliferate and differentiate into neurons at the lesion area following dorsal compression injury [19]. Focal ischemia was shown to increase the normal rate of neurogenesis in the cortex and hippocampus [20,21], and the results could be enhanced by exogenous delivery of growth factors [21]. Eventual endogenous stem cell repair responses are obviously unsatisfactory in pathology. Engineering improvements on these responses can potentially be used for therapeutic purposes.

\section{NSCs as vehicles for trophic support}

Some of the beneficial effects observed post-NSC transplantation have been attributed to trophic or anti-inflammatory support provided by NSCs. Adult NSCs transplanted into a model of chronic CNS inflammation accumulate in perivascular areas and cause apoptosis of encephalitogenic T cells [22]. C17.2 cells were shown to naturally secrete neurotrophic factors including nerve growth factor (NGF), brain-derived neurotrophic factor (BDNF), and glial cell line-derived neurotrophic factor (GDNF) [23]. Axonal growth in a SCI site following transplantation was ascribed to this effect.

\section{NSCs as vehicles for gene delivery}

Neural stem cells transplanted into the adult brain demonstrate a remarkable capacity to target sites of disease or injury [24]. This characteristic, dubbed "pathotropism", has been explored to convert NSCs into gene and drug delivery devices. GDNF-overexpressing NSCs prevented degeneration of dopaminergic neurons when transplanted into a Parkinson's disease model [25] and improved cognitive function after traumatic brain injury [25-27]. This strategy may be particularly beneficial in the targeted delivery of tumor suppressing drugs to highly invasive CNS tumors. Significant decrease in medulloblastoma tumor mass in nude mice was achieved by transplantation of a human NSC line engineered to secrete the prodrug activating enzyme cytosine deaminase [28]. Interleukin-12-secreting NSCs were shown to target glioma cells, recruit $\mathrm{T}$ cells and elicit anti-tumor immunity [29]. Tumor targeting NSCs in this study were later identified as astrocyte progenitors expressing chemokine receptor 4 [30].

\section{Biomaterials for neural stem cell delivery}

Stem cells are highly responsive to the chemical and physical state of their microenvironment. Precise engineering of microenvironmental cues using artificial materials (Table 1) can potentially control the spatial and temporal progression of regenerative processes initiated by NSC transplantation.

\section{Biomaterials and neural stem cell therapy - in vivo stud- ies}

Artificial materials can, at a first level, simply provide a physical support for the introduction of NSCs into a lesion site where a cavity has formed, such as in stroke or trauma. Polyglycolic acid (PGA), a degradable hydrogel, was used as a scaffold in the transplantation of the neural stem-like cell line C17.2 into an infarction cavity formed by the ligation of the carotid artery [31]. In this model of severe cerebral palsy, transplanted cells differentiated into neurons and astrocytes and appeared to form connections with host neurons. An implant of NSCs embedded in a blend of poly(lactic-co-glycolic acid) (PLGA) and a block copolymer of PLGA and polylysine was used in a rat hemisection model of SCI [32]. The animals exhibited motor recovery and diminished glial scar formation. Interestingly, the beneficial effects of the implant, especially regarding glial scar formation, were attributed in part to the implant material itself.

Additional levels of environmental control can be achieved by modifying the material to secrete active compounds that provide trophic support to the NSCs or to other cell types. As described above, NSCs in vitro respond strongly to single soluble factors by choosing to differentiate into predominantly one of the possible cell lineages. Biomaterial driven differentiation can be envisaged where the cell types of interest in a particular 
Table 1 A summary of some biomaterials used for transplantation of NSCs in vivo and/or controlling the characteristics of NSCs in vivo and in vitro

\begin{tabular}{lll}
\hline Biomaterials & Applications & References \\
\hline Collagen + EVAc [ethylene vinylacetate] & Factor delivery in vitro & {$[34,43]$} \\
& Culture support in vitro & \\
Alginate & Scaffold in vivo & {$[36]$} \\
PGA [polyglycolic acid] & Scaffold in vivo & {$[31]$} \\
PLGA [poly(lactic-co-glycolic acid] \pm poly-lysine & Scaffold in vivo & {$[32,33]$} \\
& Factor delivery in vivo & \\
PLLA [poly(L-lactic acid) \pm PLGA & Scaffold in vivo & {$[37,40]$} \\
& Culture support in vitro & {$[39]$} \\
ProNectin & Factor delivery in vitro & \\
& Culture support in vitro & {$[39]$} \\
PEI [polyethyleneimine] & Factor delivery in vitro & {$[41,42]$} \\
PEG [polyethylene glycol] \pm poly-lysine & Culture support in vitro & {$[44]$} \\
\hline IKVAV peptide & Culture support in vitro & \\
\hline
\end{tabular}

disease or injury situation can be produced, possibly in the desirable temporal sequence. NSCs were grown in vitro in PLGA microparticles encapsulating NGF and coated with polylysine [33]. The microparticle/NSC suspensions were then injected into the caudate/putamen of adult rats and caused elevation of NGF levels and increased NGF driven choline acetyltransferase activity in surrounding tissues. The spatial and temporal concentration profiles of NGF encapsulated in ethylene vinyl acetate (EVAc) matrix slabs in turn placed inside a collagen gel embedded with NSC or PC12 cells were described in mathematical detail [34]. Artificial extracellular matrices were generated by fusing an elastin backbone to Notch ligands, a signaling pathway heavily involved in the control of NSC state and fate [35].

The mechanical and geometrical properties of the biomaterial can be tailored to drive neurons to bridge the ends of an injury. This strategy is of particular interest in SCI therapy, where there is a clear preferred direction for axonal growth. Alginate hydrogels engineered to form parallel fibers were seeded with adult spinal cord NSCs and implanted into spinal cord lesions [36], resulting in NSCs elongating along the biomaterial fibers. Retinal precursor cells (RPCs) were seeded onto PLGA/PLLA (poly(L-lactic acid) polymers containing pores oriented normal to the plane of the scaffold [37]. RPCs delivered by the polymer/progenitor cell composite graft differentiated and cell delivery was much improved compared to injection of RPCs alone.
Biomaterials and neural stem cell therapy - in vitro studies

Polymer-based materials offer a high degree of flexibility in chemical and physical properties. Microarray technologies can be instrumental in screening for optimal materials for NSC delivery. Arrays of blends of biodegradable polyesters were tested for human mesenchymal stem cell/biomaterial interactions [38]. Cell attachment and glial differentiation of $\mathrm{C} 17.2$ cells were also tested in smaller arrays in the same study. Human NSC differentiation was tested in printed arrays of extracellular matrix molecules and signaling factors [8]. The extracellular matrix molecules laminin and fibronectin and the artificial polymers ProNectin and Polyethyleneimine (PEI) were immobilized onto arrays in combination with cytokines [39]. A comprehensive analysis was carried out to examine the fates of NSCs cultured onto the different polymer/growth factor combinations.

In vitro studies of single biomaterials for supporting NSC maintenance and differentiation have focused mostly on hydrogels. Aligned fibers of PLLA were shown to align embedded NSCs along the direction of the fibers [40]. The width of the fibers did not affect the alignment response but NSCs proliferated more on nano- than on micro-scale fibers. Polyethylene glycol (PEG) alone [41] or in combination with polylysine [42] also supported the culture of NSCs. NSC progression from proliferation to neuronal differentiation and formation of functional synapses was reported for NSCs interdispersed in Type I collagen [43]. 
A particularly innovative approach relies on the ability of certain peptides such as IKVAV [44] or RADA 16-I peptides [45] to self-assemble into nanofibers. Strikingly, IKVAV nanofibers promoted neuronal differentiation of NSCs and decreased astrocytic differentiation relative to laminin, an extracellular matrix protein containing IKVAV sequences.

\section{Conclusions}

Cell therapy using NSCs is a promising approach and the use of polymers and biomaterials can improve the outcome after transplantation in vivo as well as improve differentiation efficiency and survival in vitro. Yet there is an urgent need for the development of novel approaches based on biodegradable hydrogels, not only containing and administering "nurturing" factors but also exerting control over unwanted and disastrous outcomes, such as overgrowth and cell death. Such "second generation" polymers can very well be a crucial factor for successful implementation of neural stem cell therapy in the future.

\section{Acknowledgments}

We thank the Swedish Foundation for Strategic Research (CEDB \& OBOE), the Swedish Research Council, the Swedish Children's Cancer Foundation, and the Swedish Cancer Society (O.H.) for financial support.

\section{References}

1 Cattaneo E, McKay R. Proliferation and differentiation of neuronal stem cells regulated by nerve growth factor. Nature 1990; 347:762-765.

2 Reynolds BA, Tetzlaff W, Weiss S. A multipotent EGF-responsive striatal embryonic progenitor cell produces neurons and astrocytes. J Neurosci 1992; 12:4565-4574.

3 Stemple DL, Anderson DJ. Isolation of a stem cell for neurons and glia from the mammalian neural crest. Cell 1992; 71:973985.

4 Temple S. The development of neural stem cells. Nature 2001; 414:112-117.

5 Le Douarin NM, Creuzet S, Couly G, Dupin E. Neural crest cell plasticity and its limits. Development 2004; 131:4637-4650.

6 Johansson CB, Momma S, Clarke DL, et al. Identification of a neural stem cell in the adult mammalian central nervous system. Cell 1999; 96:25-34.

7 Reynolds BA, Weiss S. Generation of neurons and astrocytes from isolated cells of the adult mammalian central nervous system. Science 1992; 255:1707-1710.

8 Soen Y, Mori A, Palmer TD, Brown PO. Exploring the regulation of human neural precursor cell differentiation using arrays of signaling microenvironments. Mol Syst Biol 2006; 2:37.

9 Nakajima M, Ishimuro T, Kato K, et al. Combinatorial protein display for the cell-based screening of biomaterials that direct neural stem cell differentiation. Biomaterials 2007; 28:10481060 .

10 Tabar V, Panagiotakos G, Greenberg ED, et al. Migration and differentiation of neural precursors derived from human embryonic stem cells in the rat brain. Nat Biotechnol 2005; 23:601-606.

11 Flax JD, Aurora S, Yang C, et al. Engraftable human neural stem cells respond to developmental cues, replace neurons, and express foreign genes. Nat Biotechnol 1998; 16:1033-1039.

12 Englund U, Björklund A, Wictorin K, Lindvall O, Kokaia M. Grafted neural stem cells develop into functional pyramidal neurons and integrate into host cortical circuitry. Proc Natl Acad Sci U S A 2002; 99:17089-17094.

13 Pluchino S, Quattrini A, Brambilla E, et al. Injection of adult neurospheres induces recovery in a chronic model of multiple sclerosis. Nature 2003; 422:688-694.

14 Kelly S, Bliss TM, Shah AK, et al. Transplanted human fetal neural stem cells survive, migrate, and differentiate in ischemic rat cerebral cortex. Proc Natl Acad Sci U S A 2004; 101:1183911844.

15 Jeong SW, Chu K, Jung KH, et al. Human neural stem cell transplantation promotes functional recovery in rats with experimental intracerebral hemorrhage. Stroke 2003; 34:2258-2263.

16 Ogawa Y, Sawamoto K, Miyata T, et al. Transplantation of in vitro-expanded fetal neural progenitor cells results in neurogenesis and functional recovery after spinal cord contusion injury in adult rats. J Neurosci Res 2002; 69:925-933.

17 Hofstetter CP, Holmström NA, Lilja JA, et al. Allodynia limits the usefulness of intraspinal neural stem cell grafts; directed differentiation improves outcome. Nat Neurosci 2005; 8:346353.

18 Macias MY, Syring MB, Pizzi MA, et al. Pain with no gain: allodynia following neural stem cell transplantation in spinal cord injury. Exp Neurol 2006; 201:335-348.

19 Ke Y, Chi L, Xu R, et al. Early response of endogenous adult neural progenitor cells to acute spinal cord injury in mice. Stem Cells 2006; 24:1011-1019.

$20 \mathrm{Gu}$ W, Brännström T, Wester P. Cortical neurogenesis in adult rats after reversible photothrombotic stroke. J Cereb Blood Flow Metab 2000; 20:1166-1173.

21 Nakatomi H, Kuriu T, Okabe S, et al. Regeneration of hippocampal pyramidal neurons after ischemic brain injury by recruitment of endogenous neural progenitors. Cell 2002; 110:429-441.

22 Pluchino S, Zanotti L, Rossi B, et al. Neurosphere-derived multipotent precursors promote neuroprotection by an immunomodulatory mechanism. Nature 2005; 436:266-271.

23 Lu P, Jones LL, Snyder EY, Tuszynski MH. Neural stem cells constitutively secrete neurotrophic factors and promote extensive host axonal growth after spinal cord injury. Exp Neurol 2003; 181:115-129.

24 Modo M, Mellodew K, Cash D, et al. Mapping transplanted stem cell migration after a stroke: a serial, in vivo magnetic resonance imaging study. Neuroimage 2004; 21:311-317.

25 Åkerud P, Canals JM, Snyder EY, Arenas E. Neuroprotection through delivery of glial cell line-derived neurotrophic factor by neural stem cells in a mouse model of Parkinson's disease. J Neurosci 2001; 21:8108-8118.

26 Liu Y, Himes BT, Solowska J, et al. Intraspinal delivery of neurotrophin-3 using neural stem cells genetically modified by recombinant retrovirus. Exp Neurol 1999; 158:9-26. 
27 Bakshi A, Shimizu S, Keck CA, et al. Neural progenitor cells engineered to secrete GDNF show enhanced survival, neuronal differentiation and improve cognitive function following traumatic brain injury. Eur J Neurosci 2006; 23:2119-2134.

28 Kim SK, Kim SU, Park IH, et al. Human neural stem cells target experimental intracranial medulloblastoma and deliver a therapeutic gene leading to tumor regression. Clin Cancer Res 2006; 12:5550-5556.

29 Ehtesham M, Kabos P, Kabosova A, et al. The use of interleukin 12 -secreting neural stem cells for the treatment of intracranial glioma. Cancer Res 2002; 62:5657-5663.

30 Ehtesham M, Yuan X, Kabos P, et al. Glioma tropic neural stem cells consist of astrocytic precursors and their migratory capacity is mediated by CXCR4. Neoplasia 2004; 6:287-293.

31 Park KI, Teng YD, Snyder EY. The injured brain interacts reciprocally with neural stem cells supported by scaffolds to reconstitute lost tissue. Nat Biotechnol 2002; 20:1111-1117.

32 Teng YD, Lavik EB, Qu X, et al. Functional recovery following traumatic spinal cord injury mediated by a unique polymer scaffold seeded with neural stem cells. Proc Natl Acad Sci U S A 2002; 99:3024-3029.

33 Mahoney MJ, Saltzman WM. Transplantation of brain cells assembled around a programmable synthetic microenvironment. Nat Biotechnol 2001; 19:934-939.

34 Mahoney MJ, Krewson C, Miller J, Saltzman WM. Impact of Cell Type and Density on Nerve Growth Factor Distribution and Bioactivity in 3-Dimensional Collagen Gel Cultures. Tissue Eng 2006; 12:1915-1927.

35 Liu CY, Apuzzo ML, Tirrell DA. Engineering of the extracellular matrix: working toward neural stem cell programming and neurorestoration--concept and progress report. Neurosurgery 2003; 52:1154-1165; discussion 1165-1167.

36 Prang P, Muller R, Eljaouhari A, et al. The promotion of oriented axonal regrowth in the injured spinal cord by alginate-based anisotropic capillary hydrogels. Biomaterials 2006; 27:35603569.

37 Tomita M, Lavik E, Klassen H, et al. Biodegradable polymer composite grafts promote the survival and differentiation of retinal progenitor cells. Stem Cells 2005; 23:1579-1588.
38 Anderson DG, Putnam D, Lavik EB, Mahmood TA, Langer R. Biomaterial microarrays: rapid, microscale screening of polymercell interaction. Biomaterials 2005; 26:4892-4897.

39 Nakajima M, Ishimuro T, Koichi K, et al. Combinatorial display of cell-based screening of biomaterials that can direct neural stem cell differentiation. Biomaterials 2007; 28:1048-1060.

40 Yang F, Murugan R, Ramakrishna S, et al. Fabrication of nanostructured porous PLLA scaffold intended for nerve tissue engineering. Biomaterials 2004; 25:1891-1900.

41 Mahoney MJ, Anseth KS. Three-dimensional growth and function of neural tissue in degradable polyethylene glycol hydrogels. Biomaterials 2006; 27:2265-2274.

42 Ford MC, Bertram JP, Hynes SR, et al. A macroporous hydrogel for the coculture of neural progenitor and endothelial cells to form functional vascular networks in vivo. Proc Natl Acad Sci U S A 2006; 103:2512-2517.

$43 \mathrm{Ma}$ W, Fitzgerald W, Liu QY, et al. CNS stem and progenitor cell differentiation into functional neuronal circuits in threedimensional collagen gels. Exp Neurol 2004; 190:276-288.

44 Silva GA, Czeisler C, Niece KL, et al. Selective differentiation of neural progenitor cells by high-epitope density nanofibers. Science 2004; 303:1352-1355.

45 Yokoi H, Kinoshita T, Zhang S. Dynamic reassembly of peptide RADA16 nanofiber scaffold. Proc Natl Acad Sci U S A 2005; 102:8414-8419.

46 Hermanson O, Jepsen K, Rosenfeld MG. N-CoR controls differentiation of neural stem cells into astrocytes. Nature 2002; 419:934-939.

47 Johe KK, Hazel TG, Muller T, Dugich-Djordjevic MM, McKay RD. Single factors direct the differentiation of stem cells from the fetal and adult central nervous system. Genes Dev 1996; 10:3129-3140.

48 Barnabe-Heider F, Wasylnka JA, Fernandes KJ, et al. Evidence that embryonic neurons regulate the onset of cortical gliogenesis via cardiotrophin-1. Neuron 2005; 48:253-265.

49 Rajan P, Panchision DM, Newell LF, McKay RD. BMPs signal alternately through a SMAD or FRAP-STAT pathway to regulate fate choice in CNS stem cells. J Cell Biol 2003; 161:911-921. 